\title{
Effect of Battery Dynamics and the Associated Technologies on the Life Time of Wireless Sensor Networks
}

\author{
Satyanarayana Chanagala ${ }^{1}$, Mohammad Mushir Khan ${ }^{2}$, \\ ${ }^{1,2}$ Vivekananda Institute of Technology and Science (VITS), Karimnagar, Andhra Pradesh, India
}

\begin{abstract}
Battery life extension is the principal driver for energy-efficient wireless sensor network (WSN) design. However, there is growing awareness that in order to truly maximize the operating life of batterypowered systems such as sensor nodes, it is important to discharge the battery in a manner that maximizes the amount of charge extracted from it. In spite of this, there is little published data that quantitatively analyzes the effectiveness with which modern wireless sensor nodes discharge their batteries, under different operating conditions. This paper focuses on discharge profiles of battery under different conditions which could play a vital role in the life time of the wireless sensor networks. Power consumption is the limiting factor for the functionality offered by portable devices that operate on batteries. This power consumption problem is caused by a number of factors. Users are demanding more functionality, more processing, longer battery lifetimes, and smaller form factor and with reduced costs. Battery technology is only progressing slowly; the performance improves just a few percent each year. Mobile devices are also getting smaller and smaller, implying that the amount of space for batteries is also decreasing. Decreasing the size of a mobile device results in smaller batteries, and a need for less power consumption.
\end{abstract}

\section{Introduction}

Maximizing battery life is a principal objective in the design of wireless sensor networks (WSNs). This has in large part, driven the research and development of numerous technologies that aim at minimizing WSN energy consumption. However, for many electronic systems, achieved battery life is a function of not only the energy consumed by the system, but also the manner in which the system drains the battery, and specific characteristics of the battery itself. Recently, this observation has led to research in the area of "battery-driven" system design, which encompasses a set of technologies that aim at exploiting battery characteristics in order to achieve lifetime extensions beyond what can be achieved through low-power or energy-efficient design alone [1], [2]. While it is generally known that making an electronic system's discharge profile more "batteryfriendly" can help improve battery performance and hence lifetime, there is very little work that has investigated the opportunities for battery-efficient design specifically in the context of wireless sensor networks. Power consumption is the rate at which energy is consumed. With the fixed energy capacity of a battery, the power consumption directly determines the lifetime of a portable device. The challenge is doing as much as possible with the lowest amount of energy. Efficiency is the key to solve the power crisis. High performance with high power consumption does not necessarily mean less energy efficient and conversely, low performance and low power consumption does not mean that a device is more energy efficient.

The power consumption of personal portable devices is not dominated by a single component, hardware or software. Several studies have investigated the power consumption of portable devices [4]. The main conclusion is that there is no single component or single activity that dominates the power consumption in a portable device. Therefore, the power consumption of all components needs to be reduced to lower the total amount of power. Unfortunately, battery technology is not improving at the same pace as the energy requirements of handheld electronics. Therefore, energy management, once in the realm of desired features, has become an important design requirement and one of the greatest challenges in portable computing for a long time to come. Contribution of this paper is divided into two sections, first section focuses on dynamics of the battery and the second section focuses on power management techniques.

\section{Battery Dynamics}

\section{A. Understanding the electrochemistry of Lithium-ion Battery}

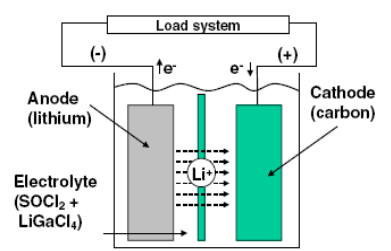

Fig.1. Schematic of a Lithium ion battery 
The standard or rated capacity of a battery which is expressed in current-time units, e.g. mAh is a measure of the total charge that can be extracted from a battery when discharged under standard load conditions. Manufacturers may specify a rated current, a constant discharge rate that corresponds to the standard load conditions. For example, a battery may be rated at $500 \mathrm{mAh}$ capacity under a rated current of $100 \mathrm{~mA}$, at $25^{\circ} \mathrm{C}$. and in this case the battery would work for 5 hours. The delivered capacity of a battery is the capacity that the battery delivers under a given load and operating environment, and is an indicator (along with battery life) of the efficiency with which the battery is discharged. Several electrochemical effects make the delivered capacity sensitive to the characteristics of the discharge profile and the environment. Hence, in practice, the delivered capacity may differ significantly from the rated capacity. The extent to which different effects are important is highly sensitive to the chemistry, type and size of the battery under consideration. Battery life depends largely on the availability of active reaction sites throughout the cathode. At low discharge currents, inactive reaction sites get uniformly distributed throughout the volume of the cathode. However, during intervals when the discharge current is large, the outer surface of the cathode gets covered with inactive sites, making many internal active sites unreachable. These rate capacity effects lead to an overall reduction in battery capacity at higher rates of discharge. When current is drawn from the battery, positively charged ions are consumed at the cathode-electrolyte interface, and are replaced by new ions that diffuse through the electrolyte from the anode. When the current drawn is sufficiently large, the rate of diffusion fails to keep up with the rate at which ions are consumed at the cathode. As a result, the concentration of positively charged ions decreases near the cathode and increases near the anode, degrading the battery's output voltage. However, if the battery is allowed to idle for a period of time, the concentration gradient decreases (due to diffusion), leading to an apparent charge recovery. As a result, the capacity and lifetime of the battery increase. The effect of ambient temperature on battery efficiency depends strongly on the specific battery chemistry being considered. Most batteries perform well at room temperature. Higher temperatures allow for increased mobility of the electrolyte materials, which result in lower internal resistance. This has the effect of increasing the effective capacity of the battery. However, continuous exposure to elevated temperatures have other undesirable effects, such as shortened cycle life that is the number of times the battery can be charged/discharged, and an increased rate of self-discharge. At lower temperatures, increased internal resistance of the battery leads to reduced capacity.

\section{B. Factors affecting Battery performance}

Many factors influence the operational characteristics, capacity, energy output and performance of a battery. The effect of these factors on battery performance is discussed in this section. When a cell or battery is discharged its voltage is lower than the theoretical voltage. The difference is caused by $I R$ losses due to cell resistance and polarization of the active materials during discharge. This is illustrated in Fig. 2. In the idealized case, the discharge of the battery proceeds at the theoretical voltage until the active materials are consumed and the capacity is fully utilized. The voltage then drops to zero. Under actual conditions, the discharge curve is similar to the other curves in Fig. 2.

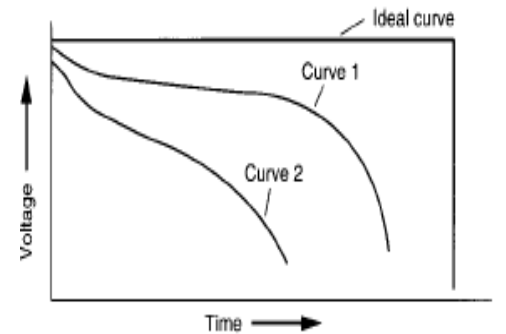

Fig.2. Characteristic discharge curves

The initial voltage of the cell under a discharge load is lower than the theoretical value due to the internal cell resistance and the resultant $I R$ drop as well as polarization effects at both electrodes. The voltage also drops during discharge as the cell resistance increases due to the accumulation of discharge products, activation and concentration, polarization, and related factors. Curve 2 is similar to curve 1, but represents a cell with a higher internal resistance or a higher discharge rate, or both, compared to the cell represented by curve 1 . As the cell resistance or the discharge current is increased, the discharge voltage decreases and the discharge show a more sloping profile.

\section{C.Current Drain of Discharge}

As the current drain of the battery is increased, the $I R$ losses and polarization effects increase, the discharge is at a lower voltage, and the service life of the battery is reduced. Fig.3.shows typical discharge curves as the current drain is changed. At extremely low current drains (curve 2) the discharge can approach the 
theoretical voltage and theoretical capacity. With increasing current drain (curves 3-5) the discharge voltage decreases, the slope of the discharge curve becomes more pronounced, and the service life, as well as the delivered ampere-hour or coulombic capacity, are reduced.

\section{Mode of Discharge (Constant Current, Constant Load, Constant Power):}

The mode of discharge of a battery, among other factors, can have a significant effect on the performance of the battery.

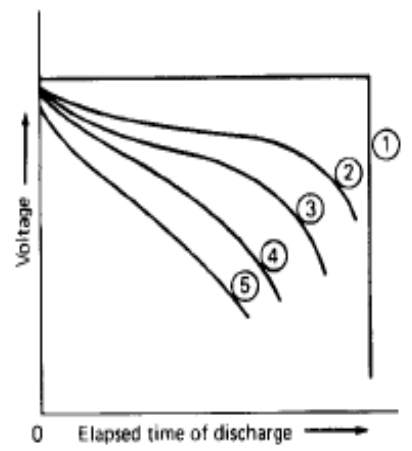

Fig.3. Battery discharge characteristics-Voltage levels

Three of the basic modes under which the battery may be discharged are:

1. Constant Resistance: The resistance of the load remains constant throughout the discharge (current decreases during the discharge proportional to the decrease in the battery voltage).

2. Constant Current: The current remains constant during the discharge.

3. Constant Power: The current increases during the discharge as the battery voltage decreases, thus discharging the battery at constant power level.

In the constant-resistance discharge mode, the current during the discharge follows the drop in the battery voltage. The power, VI or $V^{2} / R$, drops even more rapidly, following the square of the battery voltage. Under this mode of discharge, the battery discharges at a higher current than needed, draining its capacity rapidly, this will result in a shorter service life.

In the constant-current mode, the current is maintained at a level such that the power output at the cutoff voltage is equal to the level required for acceptable equipment performance. Thus both current and power throughout the discharge are lower than for the constant resistance mode. The average current drain on the battery is lower and the discharge time or service life to the end of the battery life is longer.

In the constant-power mode, the current is lowest at the beginning of the discharge and increases as the battery voltage drops in order to maintain a constant-power output at the level required by the equipment. The average current is lowest under this mode of discharge, and hence, the longest service time is obtained.

It should be noted that the extent of the advantage of the constant-power discharge mode over the other modes of discharge is dependent on the discharge characteristics of the battery.

\section{E.Temperature of Battery during Discharge}

The temperature at which the battery is discharged has a pronounced effect on its service life and voltage characteristics. This is due to the reduction in chemical activity and the increase in the internal resistance of the battery at lower temperatures. This is illustrated in Fig. 4, which shows discharges at the same current drain but at progressively increasing temperatures of the battery (T1 to $T 4$ ), with $T 4$ representing a discharge at normal room temperature.

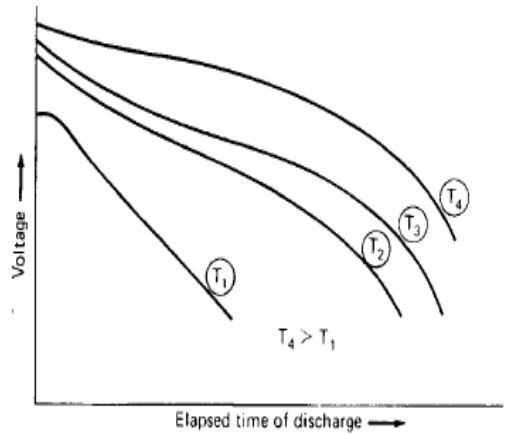

Fig.4. Effect of temperature on Battery capacity 
Lowering of the discharge temperature will result in a reduction of capacity as well as an increase in the slope of the discharge curve. Both the specific characteristics and the discharge profile vary for each battery system, design, and discharge rate, but generally best performance is obtained between 20 and $40^{\circ} \mathrm{C}$. At higher temperatures, the internal resistance decreases, the discharge voltage increases and, as a result, the ampere-hour capacity and energy output usually increase as well. On the other hand, chemical activity also increases at the higher temperatures and may be rapid enough during the discharge to cause a net loss of capacity. Again, the extent is dependent on the battery system, design and temperature.

\section{F. Type of Discharge (Continuous, Intermittent, etc.)}

When a battery stands idle after a discharge, certain chemical and physical changes take place which can result in a recovery of the battery voltage. Thus the voltage of a battery, which has dropped during a heavy discharge, will rise after a rest period, giving a sawtooth shaped discharge, as illustrated in Fig.5. This can result in an increase in service life. This improvement, resulting from the intermittent discharge, is generally greater after the higher current drains as the battery has the opportunity to recover from polarization effects that are more pronounced at the heavier loads. In addition to current drain, the extent of recovery is dependent on many other factors such as the particular battery system and constructional features, discharge temperature, end voltage, and length of recovery period.

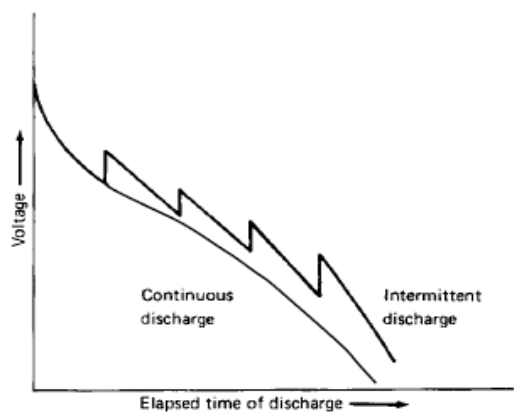

Fig.5. Effect of intermittent discharge on Battery capacity

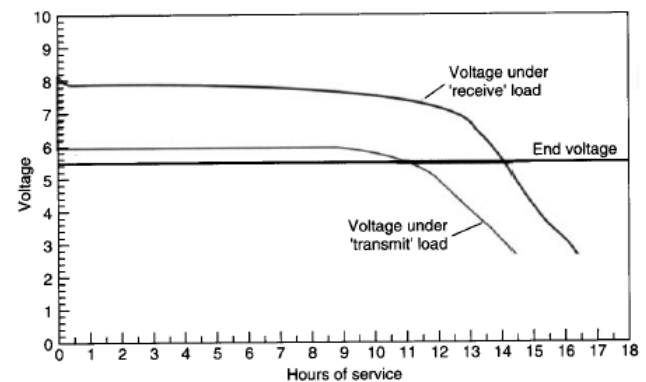

Fig.6. Typical discharge characteristics of a battery cycling between transmit and receive loads

\section{G. Duty Cycles (Intermittent and Pulse Discharges)}

Another consideration is the response of the battery voltage when the discharge current is changed during the discharge, such as changing loads from receives to transmit in the operation of a radio transceiver. Fig. 6 Illustrates a typical discharge of a radio-transceiver, discharging at a lower current during the receive mode and at a higher current during the transmit mode. Operating at two or more discharge loads is typical of most electronic equipment because of the different functions they must perform during use.

\section{A. The Elements Power Consumption}

\section{Minimizing of Power Consumption in Electronic Subsystems}

Power consumption in complementary metal-oxide-semiconductor (CMOS) ICs can be abstracted to dynamic power (switching + short) plus static power. The primary elements of both dynamic and static power are shown in the following equation:

$$
\text { Power } P(\text { avg })=P(\text { switching })+P(\text { short })+P(\text { static })
$$

Elements of Dynamic and Static Power: Dynamic power consumption arises from circuit activity and is a result of switched capacitance and short-circuits current.

$$
\text { Dynamic or Active power }=P c s+P s c
$$

Where

$$
P c s=\text { Power due to capacitive switching }=C \cdot V^{2} . F
$$


$P s c=$ Power due to short circuit current $=\mathrm{V} * \mathrm{Isc}$

$I s c=$ Short circuit current

$C=$ Capacitive load

$V=$ Supply voltage

$F=$ Switching frequency

Switched capacitance is the primary source of dynamic power consumption and arises from charging and discharging of capacitors. Psc occurs due to transition current when both the $\mathrm{N}$ channel and $\mathrm{P}$ channel are simultaneous active during switching creating a temporary short-circuit.

Static or leakage power consumption is the product of the supply voltage and the leakage current. Leakage power consumption is a result of leaking transistors and diodes and manifests itself in both active and standby modes of operation. In addition it is manufacturing process dependant.

The approach to Energy conservation is a holistic approach which includes process technology, packaging, circuit and module design, System-on-a-Chip (SoC) design, tools, and system and application software to efficiently utilize the energy available to the system.

\section{B. Nanotechnology and Nano electronics}

Material science will do the heavy lifting required to meet the use-case demands of high performance at low power consumption. Nanotechnology involves the manipulation of materials at the nanometer or the atomic scale to create structures that have novel properties and functions because of their size, shape, or composition. Nanotechnology combines existing knowledge and ongoing research at the nanometer level, with a specific emphasis on applying the science to engineering materials with enhanced and tailored properties. The continual downscaling of conventional silicon technology has reached the nanometer scale, and promises to continue this trend down to the near-molecular level. The International Technology Roadmap for Semiconductors (ITRS) has identified trends for both scaled down CMOS devices and integrated systems, and also for devices and circuit architectures based on nanoelectronic technologies. Physics and chemistry at the nanometer scale offer new opportunities for electronics and photonics, but it is also well recognized that an integrated approach is needed which includes circuits, devices, and systems.

\section{Micro-Electrical and Mechanical Systems}

MEMS are component-level devices fabricated using semiconductor-like processes etching and plating that allow for the three-dimensional " sculpting" of silicon and numerous other materials. Most MEMS devices have moving parts, but this is not an absolute requirement. The resulting products are quite diverse, ranging from sensors (inertial, pressure, fl ow, biological, chemical, and infrared) and actuators (nozzles, pumps, valves, microphones, and switches) to other novel devices, such as lab on-a-chip and arrays of mirrors. Most of the MEMS in mobile devices today fall into one of the two categories RF front-end duplexers and microphones. Other types of MEMS devices such as accelerometers for motion detection are beginning to appear in mobile devices. Future applications include navigation aids, input devices, image stabilizers, protection and security, power sources, displays, and bio-monitoring. The adoption of the MEMS solution, rather than some other approach, will be driven by three considerations: cost, physical size, and power consumption.

Radio Components: MEMS devices have two key advantages in radio applications for mobile devices: small size and linearity. The small size of MEMS components, and the resulting possibility of packaging them very close to the active circuit elements (perhaps even integrated on the same chip), minimize stray resistance, inductance, and capacitance, resulting in greater range and longer battery life. In addition, since MEMS devices tend to be more linear than semiconductor devices, replacement of a semiconductor component with a MEMS component can reduce signal distortion. The specific applications for MEMS devices in the RF section of mobile handsets include switching, filtering, matching, and signal generation (oscillators). Radio sections are the least integrated of the major mobile device sections. This is an advantage for MEMS because it is possible for designers to introduce MEMS devices as one for one replacement for specifi $\mathrm{c}$ parts without a need to completely redo multi-function integrated circuits. It also allows the possibility of changing designs at the board level to gain the full benefits of MEMS. The main advantages of MEMS implementations of RF functions are reduced signal loss, higher signal isolation, and improved linearity.

\section{Conclusions}

This paper presents discharge profiles of battery under different conditions. Power consumption is the limiting factor for the functionality offered by portable devices that operate on batteries and various aspects of this is discussed here. Discharge profiles along with the latest technologies in circuit fabrication helps in further extension of the life time of wireless sensor networks. 


\section{References}

[1]. Ralph J. Broad, "Recent Developments in Batteries for Portable Consumer Electronics Applications", Interface 8: 3, fall 1999, Electrochemical Society, Pennington, NJ.

[2]. R. Selim and P. Bro, "Performance Domain Analysis of Primary Batteries", Electrochemical Technology, J. Electrochem. Soc. 118(5) 829, (1971).

[3]. D. I. Pomerantz, "The Characterization of High Rate Batteries", IEEE Transactions Electronics, 36 (4) 954 (1990).

[4]. V. Drori, and C. Martinez, "Smart Battery Chips Maximize Usable Battery Capacity and Battery Life", Battery Power Products and Technology, April 1999.

[5]. “American National Standards Specification for Dry Cells and Batteries", ANSI C18.1M-1999, American National Standards Institute, Inc., January 1999.

[6]. Eveready Battery Engineering Data, vol. 2a, Eveready Battery Co., St. Louis, Mo.

[7]. C. Ellis. The case for higher-level power management. In Workshop on Hot Topics in Operating Systems, pp. 162, 167, 1999.

[8]. C. Chun and A. Barth. "Extreme energy conservation for mobile communications", Free scale Technology Forum, July 2006.

[9]. J. Ville and A. Vahervuori, "Energy-Awareness in Mobile and Ubiquitous Computing", Department of Computer Science, University of Helsinki, Finland.

[10]. A. Hemani, T. Meincke, S .Kumar, A. Postula, T. Olson, P. Nilsson, J. Öberg, P. Ellervee and D. Lindqvist, "Lowering power consumption in clock by using globally asynchronous locally synchronous design style", Proceedings of AC/WIEEE Design Automation Conference, 1999.

[11]. Chi-Kin Chau, Muhammad Husni Wahab, Fei Qin, Yunsheng Wang, Yang Yang, "Battery recovery aware sensor networks", $7^{\text {th }}$ International symposium on modeling, 2009

[12]. J. Redi, S. Kolek, K. Manning, C. Partridge, R. Rosales-Hain, R. Ramanathan,and I. Castineyra, "Javelen: An ultra-low energy ad hoc wireless network," Ad Hoc Networks Journal, vol. 5, no. 8, 2008.

[13]. Tokuya Inagaki and Susumu Ishihara, "HGAF: A power saving scheme for wireless sensor network", Journal of Information Processing, vol. 17, pp. 255266,Oct.2009.

[14]. Chulsung Park, Kanishka Lahiri and Anand Raghunathan, (2005) "Battery Discharge Characteristics of Wireless Sensor Nodes",An Experimental Analysis'publication in the IEEE SECON 2005, pp. 430 - 440.

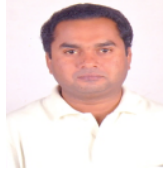

Satyanarayana Chanagala received his special diploma in Electronics with specialization in Communication Engineering from Government Institute of Electronics, Secunderabad, Andhrapradesh, India in 1992.As the part of the course he has undergone six months industrial training in Hindustan Aernautics limited, Avionics division, Hyderbad.During the same he has won the National merit scholarship from government of India. He received Bachelors degree in Electronics Engineering from Nagpur University, Maharashtra, India, securing $15^{\text {th }}$ position in the University examination in 1997 and got his Masters degree in Technology in the specialization of Semiconductor Lasers and Fiber Optic Systems, from Indian Institute of Technology, Kanpur, Uttarpradesh, India. He was a Lecturer, Sr.Lecturer, Assistant Proffesor with department of Electronics Engineering of KITS, Ramtek, Maharashtra, India.He was Associate Proffesor and Proffesor, ACE Engineering college and VITS, Karimnagar, Andhrapradesh, India.He published 12 number of papers in Inernational Journals and Conferences. His research interests include Digital Image Processing, Digital Signal Processing and wireless-sensor networks. At present he is engaged in "Life time extension of wireless sensor networks" as a part of his Doctoral thesis. 